\title{
Políticas públicas na Hanseníase: contribuição na redução da exclusão social
}

Public policies in Leprosy: contribution in reduction of social exclusion

La política pública en la Lepra: contribución en la reducción de la exclusión social

Regina Célia Dias', Elisete Silva Pedrazzani ${ }^{1}$

'Universidade Federal de São Carlos. Departamento de Enfermagem. São Carlos, SP

Submissão: 03/09/2008

Aprovação: 23/10/2008

\section{RESUMO}

O estudo analisa o desempenho do setor de hanseníase de um município do estado de Minas Gerais, no período de janeiro de 1996 a dezembro de 2004, por meio de um estudo descritivo e retrospectivo dos prontuários dos pacientes Que iniciaram poliQuimioterapia neste período. Verificou-se a freqüente presença da supervisão técnica estadual, com atendimento aos casos mais graves, cursos de capacitação aos profissionais e o constante apoio frente às dificuldades. $\mathrm{O}$ resultado foi o aumento do número de casos detectados e a melhoria na Qualidade do atendimento ao portador de hanseníase. Atualmente, 100\% dos casos são avaliados Quanto ao grau de incapacidade, além do monitoramento das reações hansênicas e neurites, prevenindo assim as deformidades.

Descritores: Hanseníase; Políticas de saúde; Prevenção \& controle.

\section{ABSTRACT}

The study examines the performance of the sector of leprosy as a municipality of Minas Gerais, from January 1996 to December 2004 , through a descriptive and retrospective study of medical records of patients who started multi-drug therapy in this period. It was the freeuent presence of the state technical supervision, with attention to the most severe cases, courses of training for professionals and constant support in the face of difficulties. The result was increasing the number of cases detected and improvement in Quality of care to the diseased of leprosy. Currently, 100\% of cases are assessed on the degree of disability, in addition to monitoring the reactions and neuritis, thus preventing deformities.

Descriptors: Leprosy; Health policy; Prevention \& control.

\section{RESUMEN}

El estudio examina el desempeño del sector de la lepra de un municipio de estado de Minas Gerais, a partir de enero de 1996 a diciembre de 2004, a través de un estudio descriptivo y retrospectivo de historias clínicas de los pacientes que iniciaron poli Quimioterapia en este período. Fue verificada a frecuente presencia de la supervisión técnica del Estado, con atención a los casos más graves, cursos de formación para los profesionales y apoyo constante ante las dificultades. El resultado fue el aumento del número de casos detectados y la mejora en la calidad de la atención al portador de la lepra. Actualmente, el 100\% de los casos son evaluados por el grado de discapacidad, además de supervisar las reacciones y neuritis, evitando así deformaciones.

Descriptores: Lepra; Política de salud; Prevención \& control. 


\section{INTRODUÇÃO}

O Brasil ocupa hoje o segundo lugar mundial em número de casos de hanseníase. Nos últimos anos foram elaborados planos, com metas e estratégias para se alcançar a eliminação da hanseníase, isto é, chegar a uma taxa de prevalência menor Que I caso por 10.000 habitantes. Conseguiu-se a redução da taxa de prevalência e o aumento do número de casos tratados com a polieuimioterapia (PQT).

Porém, apesar dos esforços, a meta de eliminação para o país ainda não foi atingida. Dentre os fatores Que impediram alcançar a meta está a permanência de casos não diagnosticados, prevalência oculta, responsáveis pela manutenção de fontes de contágio na população. É preciso Quebrar a cadeia de transmissão da doença por meio do diagnóstico precoce de todos os casos e tratamento imediato com a polieuimioterapia ${ }^{(1)}$.

Estudo realizado pela Área Técnica de Hanseníase do estado de Minas Gerais em 2000, mostrou Que o estado vinha deixando de diagnosticar, em média, 1.000 casos a cada ano ${ }^{(1)}$.

Dados do Ministério da Saúde (1998-2000) mostram um aumento no número de casos principalmente na região Norte e Nordeste (56\% dos casos), enQuanto apenas $5 \%$ são da região sul do país. Coeficientes de prevalência para as regiões sul e sudeste chegam a ser 15 e 10 vezes menores, respectivamente, Quando comparados a outras regiões ${ }^{(2)}$.

O Estado de Minas Gerais ocupava em 1991, o primeiro lugar no Brasil em número de casos. Com o objetivo de atingir a meta de eliminação da Hanseníase elaborou-se o "Plano de Emergência para Controle da Hanseníase" (1991 a 1994) e o "Plano de Eliminação da Hanseníase em Minas Gerais" (1995 a 2000). Estes planos priorizaram a capacitação dos trabalhadores dos serviços de atendimento ao portador de hanseníase, buscando o diagnóstico precoce, o aumento dos casos tratados com a polieuimioterapia e a redução da taxa de abandono. O resultado foi uma Queda na prevalência de 22,2 casos/10.000 habitantes em 1991 para 3,2 casos/ 10.000 habitantes em $2000^{(3)}$.

Com a diminuição da prevalência da hanseníase e terapias mais breves, os profissionais das unidades básicas de saúde são capazes de tratar o portador de hanseníase sem que isso implique uma sobrecarga no trabalho dos mesmos ${ }^{(4)}$. Assim, em 2002, o estado preconiza a Descentralização dos serviços de Hanseníase, com uma proposta de hierarQuização do atendimento ${ }^{(3)}$.

A integração dos programas de controle da hanseníase na rede básica de saúde é considerada atualmente a melhor estratégia para eliminação da doença, para o diagnóstico precoce e melhoria na Qualidade do atendimento ao portador da hanseníase, facilitando o acesso ao tratamento, a prevenção de incapacidades e a diminuição do estigma e da exclusão social ${ }^{(5)}$.

A implantação de um programa integrado é complexo e se realizado à revelia poderá comprometer o sistema de vigilância e a Qualidade da assistência ao doente. Por isso, o planejamento deve ser cuidadoso e adeQuado à situação local(6).

No Brasil, os peeuenos municípios enfrentam dificuldades comuns na administração da saúde pública. As mudanças políticas locais e a constante inversão de prioridades a cada novo governo têm como conseQüência a desestruturação de alguns serviços e programas de saúde; aliado a isso está a falta de profissionais Qualificados. Neste contexto, a manutenção dos serviços de hanseníase, assim como de outros programas de saúde, reQuer um esforço contínuo de todos os profissionais envolvidos.

O estudo foi realizado no município de São Sebastião do Paraíso, situado no sudoeste do estado de Minas Gerais, com uma população de 58.335 habitantes no ano $2000^{(7)}$, e Que apresenta uma alta taxa de incidência de casos de hanseníase.

O objetivo foi analisar a incidência de casos no município e avaliar a Qualidade do atendimento ao portador de hanseníase.

\section{METODOLOGIA}

Foi realizado um estudo descritivo e retrospectivo dos prontuários dos usuários Que iniciaram poliQuimioterapia no município, no período de janeiro de 1996 a dezembro de 2004.

Para cada ano foram levantados: o número de casos novos; as formas clínicas, classificadas em paucibacilar e multibacilar; a distribuição por gênero; a distribuição por faixa etária e a avaliação do grau de incapacidade no início e no final do tratamento.

Também levantou-se em Que momentos os técnicos da Secretaria de Estado da Saúde estiveram presentes na unidade, ministrando cursos de capacitação ou atendendo aos usuários.

Os dados coletados foram registrados em planilhas, utilizandose o programa Excel 97 SR-2, para a análise estatística.

\section{RESULTADOS E DISCUSSÃO}

A partir de 1998, há um aumento no número de casos detectados, coincidindo com os momentos de intensificação da presença da supervisão técnica estadual, por meio de cursos de capacitação e acompanhamento de casos (Gráfico l).

Acontece uma mudança na classificação dos casos Quanto a sua forma clínica. Até o ano de 1997 a maioria dos casos de hanseníase do município era classificada de maneira equivocada como paucibacilares, resultando em tratamentos ineficazes. Uma capacitação adeeuada dos profissionais aliada a um acompanhamento dos casos junto aos supervisores da equipe técnica estadual, possibilitou uma classificação correta no diagnóstico e uma revisão dos casos, sendo Que muitos foram retratados. Em 2004 os casos paucibacilares começam a crescer, agora de maneira mais confiável, sinalizando um aumento no diagnóstico precoce (Gráfico 2). Cresce também o número de casos suspeitos encaminhados pela rede de atenção básica em saúde.

Em 2002, a equipe da unidade de atendimento em hanseníase assume a capacitação no município, com vistas a uma futura descentralização do atendimento. Inicia-se a capacitação dos auxiliares de enfermagem da rede básica de saúde, com 8 horas de teoria e um estágio de 16 horas, no setor de atendimento em hanseníase, experienciando situações de diagnóstico, tratamento, surtos reacionais e prevenção de incapacidades.

Historicamente de 1997 a 2002 o município sempre teve mais portadores de hanseníase do sexo masculino. No ano de 1998 , cerca de $68 \%$ dos casos eram do sexo masculino para $32 \%$ do sexo feminino. Em 2003 e 2004 este Quadro se inverte e as mulheres passam a ser em maior número, correspondendo a $54 \%$ de casos femininos e $46 \%$ masculinos. Esta mudança coincide com a capacitação de funcionários da atenção básica, Que ampliou o acesso ao diagnóstico a esta população. 


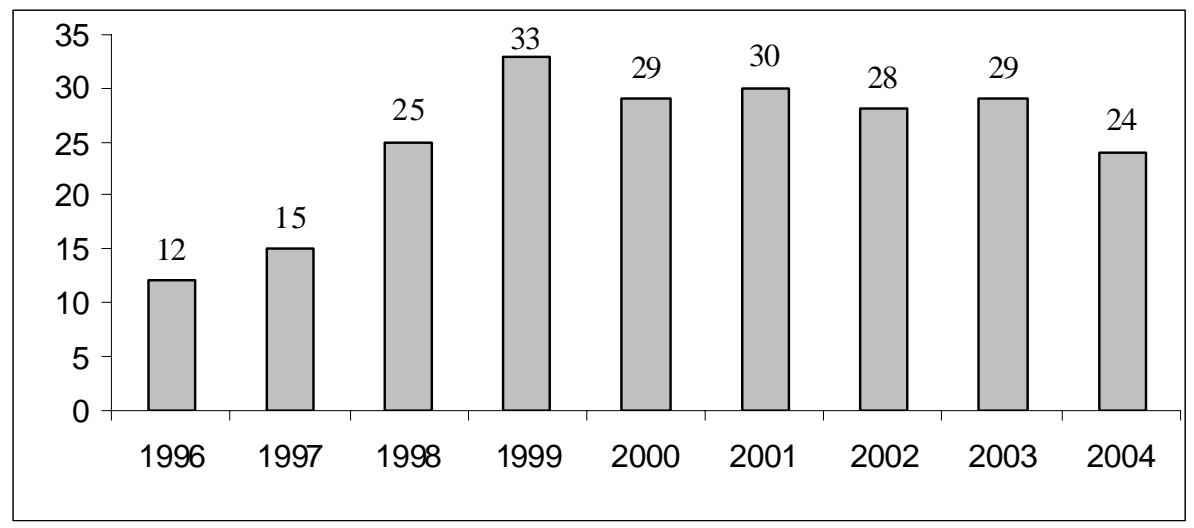

Gráfico 1. Número de casos novos Que iniciaram PQT de 1996 a 2004.

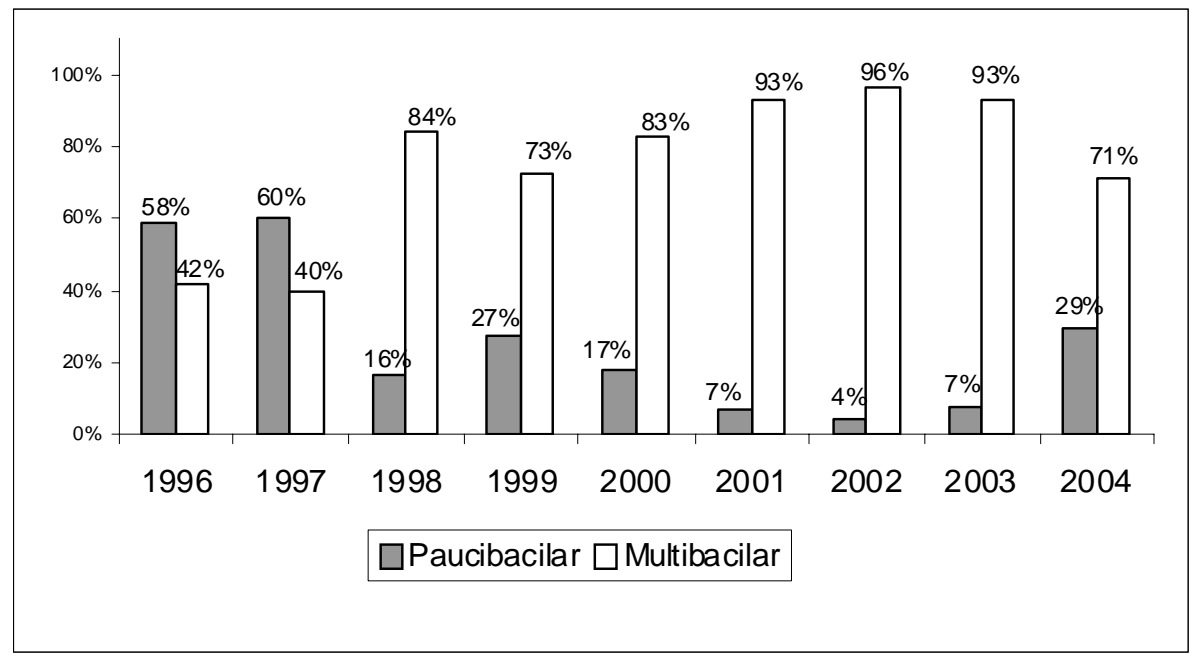

Gráfico 2. Distribuição da classificação dos casos novos Quanto à forma clínica.

Até 1997 não eram desenvolvidas ações de prevenção de incapacidades, pela falta de profissionais capacitados; portanto essa etapa de capacitação iniciou-se no ano de 1998, o Que contribuiu significativamente para a melhoria na Qualidade do atendimento ao portador de hanseníase com a realização de ações de prevenção durante todo o tratamento.

As incapacidades constituem o principal problema decorrente da hanseníase. É importante identificar os fatores de risco envolvidos, de modo a acompanhar os pacientes mais propensos com maior atenção ${ }^{(8)}$.

Muitos doentes já apresentam incapacidades na fase de diagnóstico e outros as desenvolvem durante o tratamento. Além disso, todos os doentes com perda ou diminuição da sensibilidade encontram-se em risco de desenvolver incapacidades adicionais, o Que torna essencial a prevenção das mesmas por profissionais treinados nos serviços de saúde.

Prevenção de incapacidades são medidas Que visam evitar a ocorrência de danos físicos, emocionais, espirituais e sócioeconômicos. No caso de danos já existentes, a prevenção significa a adoção de medidas com o objetivo de evitar as complicações ${ }^{(9)}$.

As tentativas de sistematizar o estudo das incapacidades geradas pela hanseníase levaram ao desenvolvimento pela OMS de um formulário para anotações das incapacidades em cada paciente, considerando três graus de incapacidade, em ordem crescente, segundo o acometimento de mãos, pés e olhos. O grau zero corresponde à ausência de incapacidades devido à hanseníase e os graus 1 e 2 decorrem de alterações sensitivas e/ou motoras de gravidade crescente. $\mathrm{O}$ grau 2 inclui as lesões e deformidades mais severas $^{(10)}$

Os portadores de hanseníase, ainda hoje, sofrem com o estigma da doença. Entre os motivos de discriminação encontram-se a falta de informação sobre a doença, a incapacidade física e as deformidades causadas pelo comprometimento dos nervos periféricos.

A partir de 2001, 100\% dos usuários Que iniciaram tratamento no município passam pela prevenção de incapacidades. Os casos com incapacidades mais severas caíram de 24\% em 1998 para 13\% em 2004 , eneuanto os de grau zero passaram de $24 \%$ para $62 \%$ no mesmo período (Gráfico 3).

\section{CONSIDERAÇÕES FINAIS}

O preconceito Que permeia a vida do portador de hanseníase e, portanto as dificuldades encontradas para inserção no seu meio social estão diretamente relacionadas à adoção de práticas inclusivas pelos 

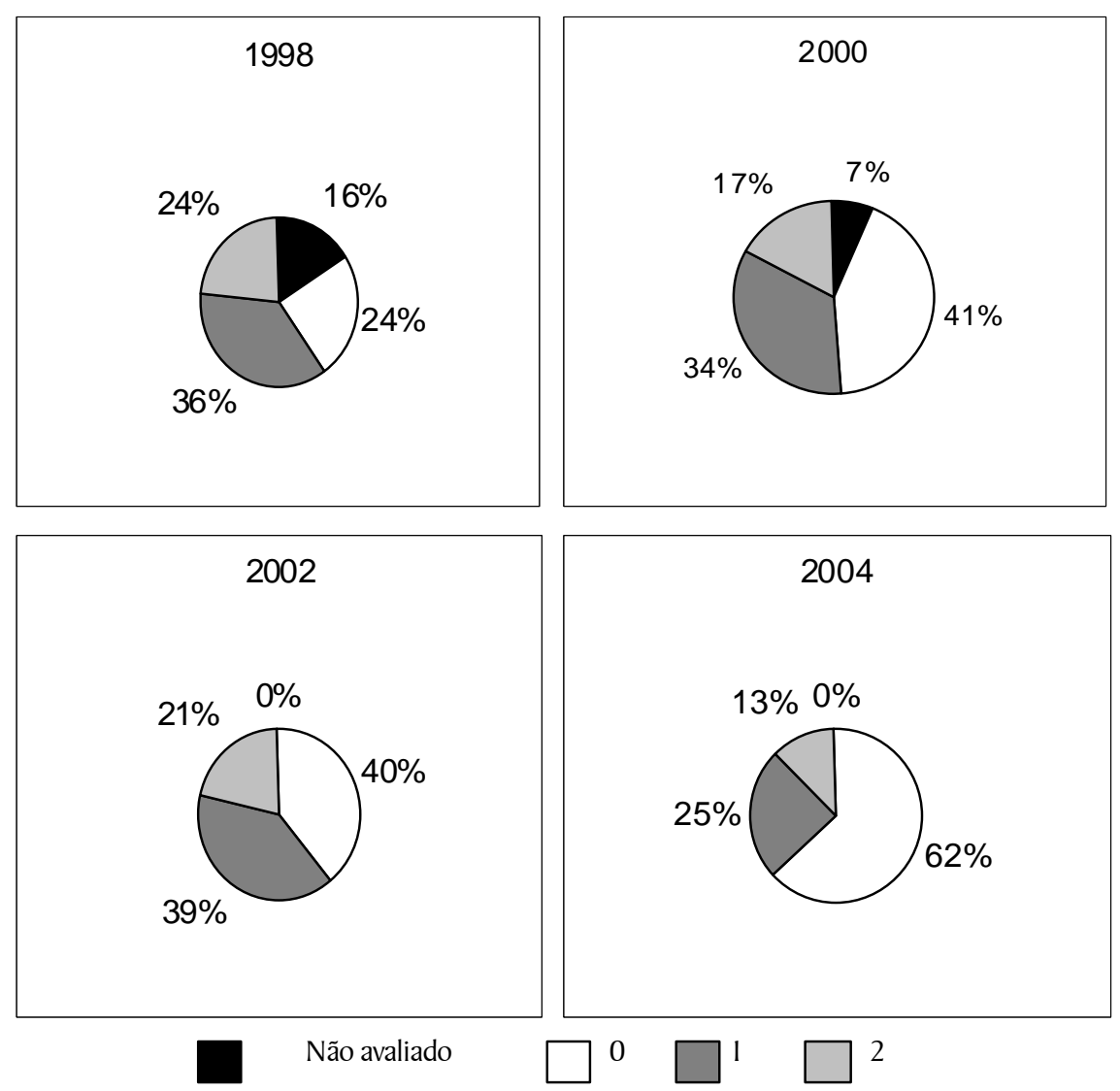

\section{Gráfico 3. Avaliação do Grau de Incapacidade dos casos novos Que iniciaram PQT nos anos 1998, 2000, 2002 e 2004.}

serviços de saúde e de ações diretas visando o diagnóstico precoce e a prevenção de incapacidades ao portador de hanseníase.

As políticas públicas de saúde implantadas pelo estado de Minas Gerais no setor de hanseníase, por meio da educação permanente, transformaram a realidade local: baixando a prevalência da doença, reduzindo a taxa de abandono, ampliando o acesso ao tratamento e melhorando a Qualidade de atendimento, contribuindo na redução da exclusão social dos portadores de hanseníase.

\section{REFERÊNCIAS}

1. Secretaria de Estado da Saúde (MG). Avaliação das ações de controle da hanseníase em Minas Gerais no ano de 2000. In: Seminário Estadual de Hanseníase. 2001 maio 7-8; Belo Horizonte (MG), Brasil. Belo Horizonte (MG): Secretaria de Estado da Saúde de Minas Gerais; 2000.

2. CONASENS: uma trajetória de luta na aceleração das ações para eliminação da hanseníase no Brasil. J Conasems 200 I; 5 (78): 8.

3. Secretaria de Estado da Saúde de Minas Gerais. Área Técnica de Hanseníase. Estratégias para eliminação da hanseníase em Minas Gerais até o ano 2005. In: Seminário Estadual de Hanseníase. 2002 out 17-18; Belo Horizonte (MG), Brasil. Belo Horizonte (MG): Secretaria do Estado da Saúde; 2002.

4. Visschedijk I, Engelhard A, Lever P, Grossi MAF, Feenstra P. Leprosy control strategies and the integration of health services: an international perspective. Cad Saúde Pública 2003; 19(6): 1567-81.
5. Feenstra P, Visschedijk I. O controle da hanseníase pelos serviços gerais de saúde - renascimento do conceito de integração. Leprosy Rev 2002; (esp): 47-57.

6. Soutar D. Integração dos serviços de lepra. Leprosy Rev 2002; (esp): 45-6.

7. Instituto Brasileiro de Geografia e Estatística (BR). Censo 2000. Rio de Janeiro: IBGE; 2000.

8. Pimentel MIF, Borges E, Sarno EN, Nery IAC, Gonçalves RR. O exame neurológico inicial na hanseníase multibacilar: correlação entre a presença de nervos afetados com incapacidades presentes no diagnóstico e com a ocorrência de neurites francas. An Bras Dermatol 2003; 78(5): 561-8.

9. Virmond M, Vieth H. Prevenção de incapacidades na hanseníase: uma análise crítica. Med Ribeirão Preto 1997; 30: 358-63.

10. Ministério da Saúde (BR). Secretaria de Políticas Públicas. Área Técnica de Dermatologia Sanitária. Manual de Prevenção de Incapacidades. Brasília: Ministério da Saúde; 2001. 